\title{
Habilidades cognitivas, autoeficacia y estrategias de aprendizaje: indicadores y determinantes del rendimiento académico en el alumnado de educación
}

\section{secundaria}

\author{
Determinants of academic performance in secondary school students and indicators: cognitive \\ skills, self-efficacy and learning strategies
}

\author{
Eduardo Barca-Enríquez, Juan Carlos Brenlla, Manuel Peralbo, Leandro S. Almeida*, Ana Mª Porto y Alfonso Barca \\ Universidade da Coruña y *Universidade do Minho
}

\begin{abstract}
Resumen
Con este trabajo se analiza la capacidad predictiva de variables cognitivas en interacción con las de autoeficacia y de estrategias de aprendizaje sobre el rendimiento académico medio del alumnado de Educación Primaria y Secundaria de Galicia y Norte de Portugal. La muestra era de 801 alumnos de $7^{\circ}$ de Enseñanza Básica (Portugal) y de $1^{\circ}$ de ESO (España) y del total de la muestra 385 eran del norte de Portugal (48\% varones y 52\% mujeres), de edades comprendidas entre 10 y 16 años, (media de 12,4 años y desviación típica 0,94 años) y 416 de Galicia (España) (51\% varones y 49\% mujeres), de edades comprendidas entre 11 y 15 años, (media de 12,3 años y desviación típica 0,70).

Los resultados apuntan a que son las variables cognitivas relacionadas con la capacidad de utilización de razonamiento con símbolos lógiconuméricos y razonamiento abstracto y verbal las que mantienen una alta correlación con el Rendimiento. Del mismo modo, la autoeficacia, entendida como la capacidad percibida para la realización de cualquier tarea y las estrategias de organización y comprensión mantienen una correlación alta y significativa con el rendimiento académico. Se ha comprobado también que la interacción de dichas variables tienen una alta capacidad predictiva sobre el buen rendimiento académico del alumnado de educación secundaria a excepción de las Estrategias superficiales que mantiene una relación negativa con el rendimiento.
\end{abstract}

Palabras clave: habilidad cognitiva, autoeficacia, estrategia de aprendizaje, rendimiento académico

La inteligencia ha sido uno de los constructos psicológicos sobre el que, quizás, se ha investigado en mayor medida a lo largo de la historia de la psicología.
Hoy ya hablamos con mayor profusión de habilidades cognitivas, términos que amplían el constructo de inteligencia y la diversifican. Sin duda todavía sigue siendo uno de los núcleos temáticos especialmente relevantes en la psicología actual. Más importancia alcanza este constructo cuando se le implica directamente con las situaciones educativas $\mathrm{y}$, en concreto, en los procesos de enseñanza y aprendizaje escolar. De hecho la inteligencia surge con fuerza en la psicología a partir de los trabajos de Binet y Simon cuando crean precisamente el modo de medirla y cuantificarla a través de su famoso test o escala psicológica que por primera vez crean y ya se utiliza a principios del siglo XX con la finalidad de obtener un cociente como resultado de la aplicación de diferentes pruebas que a su vez se promedian resultando el ya famoso cociente intelectual, el denominado CI. Este hecho ha tenido un enorme impacto en Europa y posteriormente en Estados Unidos. A partir de ahí se ha dado un desarrollo extraordinario al tema de la inteligencia y su aplicabilidad a diferentes contextos sociales, destacando sobre todos, los educativos. Los trabajos de Howard Gardner a partir de la década de los 80 ha apuntando a las inteligencias múltiples, por lo que sustituimos dicha terminología por la de habilidades cognitivas que se aproximan con mayor exactitud a lo que se quiere expresar con estos constructos.

En el contexto escolar actualmente se aplican las pruebas psicológicas de análisis de la de las habilidades cognitivas con la finalidad, preferentemente, de tratar de conocer como ocurre la estructura de la inteligencia y el desarrollo cognitivo de los alumnos, aparte de obtener alguna información útil sobre las posibles dificultades de aprendizaje que pueden ocurrir en dichos alumnos (Lemos, Almeida, Guisande y Primi, 2006) porque, precisamente Binet y Simon, en Francia, han sido los primeros autores que han creado el llamado cociente de inteligencia que, por otra parte tenía, básicamente, la 
finalidad de diferenciar a aquellos alumnos que tenía discapacidades cognitivas o dificultades de aprendizaje. Con ello lo que se había logrado era la implicación de las funciones cognitivas básicas (percepción, atención y memoria) y superiores (razonamiento, comprensión, relación y evaluación) con los rendimientos escolares, es decir, con lo que hoy se entiende como rendimiento académico de los alumnos.

Inteligencia o habilidades cognitivas y estrategias de aprendizaje

En la actualidad, con una perspectiva histórica todavía corta pero fecunda, creemos de acuerdo con Almeida y otros investigadores (Almeida, 1985, 1992; 2003, 2006; Spinath, Spinath, Harlaar y Plomin, 2006; Sternberg, Grigorenko y Bundy; 2001), que el concepto de inteligencia permanece como un aspecto fundamental en el contexto educativo y se define como la capacidad de los individuos para aprender. Parece que se trata de una definición recurrente, ya que tanto los autores factorialistas de la década de los años cincuenta y sesenta como Vernon, Spearman, Thurstone, y algo más tarde Guilford, así como toda la línea piagetiana insisten mucho en que inteligencia hay que asociarla a la capacidad de los individuos para adaptarse al medio y ello implica el despliegue de habilidades y aptitudes ante y sobre el medio ambiente en el que los sujetos conviven y se desarrollan. Por eso, a partir del descubrimiento del CI por Binet y Simon a principios del siglo $\mathrm{XX} \mathrm{y}$, posteriormente confirmado por Wechsler y otros autores, no se ha dejado de relacionar de modo empírico el concepto de inteligencia, y sus diferentes dimensiones que se iban descubriendo, con los resultados escolares, con las notas obtenidas, o con el también llamado rendimiento académico. Incluso se han relacionado las capacidades intelectuales o cognitivas con la edad de los sujetos y con frecuencia con la extensión de la escolaridad de los alumnos (Barca y Peralbo, 2002).

Actualmente se sabe que la inteligencia y sus dimensiones o habilidades cognitivas están muy moldeadas por las experiencias educativas de los sujetos (Ackerman, 1996; Almeida, 1985, 1988, 1996; 1887; 2003; Almeida, Antunes, Martins y Primi, 1997; Cattel, 1971; Ceci, 1991; Primi y Almeida, 2000) y en concreto por sus vivencias, por una parte, familiares pero, fundamentalmente por las vivencias escolares, ya que pensemos que los alumnos permanecen en las escuelas alrededor de unos 12-14 años con lo cual supone un tiempo lo suficientemente largo y denso para la sedimentación de experiencias educativas y escolares con un peso muy importante a lo largo de la historia personal de los alumnos.

Por otra parte, la autoeficacia es en esencia una capacidad percibida por el estudiante para abordar con éxito sus tareas de estudio y aprendizaje. La autoeficacia académica percibida se define como los juicios personales de las propias capacidades para organizar y ejecutar cursos de acción que conducen a tipos de acciones educativas designadas (Bandura, 1982; 1999; Schunk, 1989). Bandura postulaba que las creencias de eficacia influyen sobre el nivel de esfuerzo, persistencia y selección de actividades. Los estudiantes con un alto sentido de eficacia para completar una tarea educativa participarán con mayor disposición, se esforzarán más y persistirán durante más tempo que aquellos que dudan de sus capacidades ante las posibles dificultades.

En las situaciones educativas de los procesos de enseñanza y aprendizaje, la tarea principal que debe llevar a cabo el alumnado es, en un sentido amplio, aprender antes, durante y después de participar en las distintas actividades que se realizan cuando se abordan tareas escolares y, también sabemos que son las propias actividades de estudio las que más tiempo ocupan a los alumnos. De acuerdo con Hernández y García (1991), el estudio es una modalidad de aprendizaje, una situación específica de actividad académica de carácter cognitivo y metacognitivo, frecuentemente individual e interactiva, organizada, estructurada e intencional, intensiva, autorregulada y fundamentada, habitualmente, en unos materiales escritos, en un texto y que, además, crea expectativas, automotivación, genera autoconceptos y supone siempre un esfuerzo personal (Garma y Elexpuru, 1999).

Sin embargo, para llevar a cabo las tareas de estudio es preciso desarrollar, entre otros tipos de mecanismos cognitivos complejos, aquellas habilidades específicas que generen estrategias y técnicas de aprendizaje. Es decir, para llevar a cabo las diferentes tareas de estudio los alumnos deben adquirir, procesar, recuperar y transferir con eficacia la información, siendo de gran ayuda para este fin, el uso de estrategias de aprendizaje. La definición y categorización de las estrategias ha sido abordada por diversos autores (Dansereau, 1978; Nisbet y Shucksmith, 1987; Román y Gallego, 1991; 1997; Beltrán, 1993; Beltrán, Perez y Ortega, 2006; Cano, 1987, 1993; Cano y Justicia, 1993; Justicia y Cano, 1993). De acuerdo con estos autores citados anteriormente, una definición integradora de estrategias de aprendizaje pasa por considerarlas como aquellos mecanismos de control de los que dispone el sujeto para dirigir sus modos de procesar la información y facilitar, así, la adquisición de información, el almacenamiento y recuperación de la información.

En líneas generales, los autores están de acuerdo en considerar que las estrategias cognitivas, metacognitivas y de apoyo al aprendizaje, también denominadas estrategias de procesamiento de aprendizaje, son conductas que desarrolla el sujeto que aprende para operar sobre el modo en que la información es procesada, codificada y recuperada para el logro de su posterior aplicabilidad y transferencia. Implican secuencias integradas de procedimientos o tareas y habilidades mentales que se activan con el propósito de facilitar la adquisición, almacenamiento y/o utilización de la información. Se trata de competencias necesarias para que un aprendizaje sea efectivo, incluyendo las estrategias y habilidades que los estudiantes necesitan para manejar y controlar su propio aprendizaje en distintas circunstancias (Barca, Porto, Santorum, Morán y Brenlla, 2008; Barca, Almeida, Porto, Peralbo y Brenlla, 2012).

En definitiva, el objetivo o meta del aprendizaje consiste en adquirir conocimientos y llegar a la 
comprensión. La adquisición de conocimientos y la comprensión se desarrollan a través de procesos interactivos de enseñanza y estudio. Los profesores, en la actualidad, son conscientes de que el aprendizaje ya no consiste en un simple proceso de adquisición de fragmentos de datos o información, sino que reconocen que los alumnos tienen sus propias pautas personales de conducta más o menos estables (personalidades), sus motivaciones, sus experiencias y percepciones y que, éstas poseen siempre algún tipo de repercusión sobre el aprendizaje. Por tanto, el aprendizaje va a estar mediatizado por una serie de variables que es preciso conocer para su adecuado tratamiento con el fin de lograr el objetivo de que el aprendizaje ocurra realmente en las mejores condiciones posibles (Barca, Marcos, Núñez, Porto y Santorum, 1997; Barca, Almeida, Porto, Brenlla, Barca, Morán, 2010).).

En este trabajo, aparte de tratar de analizar la importancia y capacidad predictiva que ya sabemos que posee la inteligencia, en cuanto habilidades cognitivas, sobre el rendimiento académico de los alumnos, queremos también analizar cómo se comportan otras dimensiones de la conducta de estudio como son la autoeficacia entendida como la capacidad percibida para la realización de cualquier tarea y las estrategias de aprendizaje en interacción con las habilidades cognitivas sobre el propio rendimiento académico

Método
Muestra
Integrada por un total de 801 alumnos de $7^{\circ}$ de
Enseñanza Básica (Portugal) o de $1^{\circ}$ de ESO (España).
Del total de la muestra 381 eran del norte de Portugal
(48\% varones), de edades comprendidas entre 10 y 16
años, (media de 12,4 años y desviación típica 0,94 años)
y 404 de Galicia (España) (51\% varones), de edades
comprendidas entre 11 y 15 años, (media de 12,3 años y
desviación típica 0,70). Su procedencia es de tipo
urbano, confirmando que la mayoría procede de la
periferia de ciudades como Porto, Guimaraes, Braga y
Chaves, así como de A Coruña, Ourense y Santiago de
Compostela (ver tabla 1),

\section{Instrumentos}

En cuanto a la medida de la inteligencia contamos en este trabajo con la excelente Escala o Bateria de Raciocinio de Leandro Almeida (BPRD-7/9), elaborada por el autor (Almeida, 1986, 1988) y publicada en la Universidade do Porto en 1986 y Universidade do Minho en 2003. Hay otra edición del año 1997 de esta Batería publicada por la Casa do Psicologo de Sao Paulo. Está compuesta por cinco subtests: Razonamiento Abstracto (RA), Razonamiento Verbal (RV), Razonamiento Numérico (RN), Razonamiento Espacial (RE) y Razonamiento Mecánico (RM). Se fundamenta en las concepciones factoriales de la inteligencia más recientes a las que nos heme referido con brevedad anteriormente. En concreto esta Batería evalúa, por una parte, las capacidades o habilidades de aprehensión y aplicación de relaciones conceptuales (razonamiento) $\mathrm{y}$, por otra se tiene en cuenta la especificidad de contextos o contenidos a los que se pueden aplicar tales habilidades de razonamiento
(Lemos, Almeida, Guisande, 2006). Con esta Batería BPDR se han realizado numerosas investigaciones en Portugal $y$ en Brasil principalmente con muestras de alumnado de educación secundaria pensando en contribuir al análisis de las dificultades de aprendizaje y en la orientación vocacional y, por lo tanto, se dispone de una amplia base de datos para la verificación de sus propiedades psicométricas, especialmente de su validez y fiabilidad, cuyos coeficientes se muestran óptimos a través de diferentes investigaciones realizadas al efecto (Almeida, 1985; Almeida, Antunes, Martins y Primi, 1997; Almeida y Campos, 1986; Almeida, Candeias, Primi, Ramos, Gonçalves, Coelho, Dias, Miranda y Oliveira, 2003; Primi y Almeida, 2000; Primi, Almeida y Lucarelli, 1996).

Tabla 1.

Datos de la Muestra y Medias y desviaciones típìcas de las variables independientes ( $R A, R V, R N)$ Subescala de Estrategias de Aprendizaje y Autoeficacia (EAE): Estrategias de organización y comprensión (EOC) Autoeficacia (AE), Estrategias superficiales (ES) y variable dependiente (Nota media global-NMG) del alumnado de Educación secundaria de Galicia y Norte de Portugal

\begin{tabular}{lccccc}
\hline & \multicolumn{2}{c}{$\begin{array}{c}\text { Portugal } \\
(\mathrm{N}=381)\end{array}$} & & \multicolumn{2}{c}{ Galicia/España } \\
& \multicolumn{2}{c}{$(\mathrm{N}=404)$} \\
& media & $\mathrm{dt}$ & & media & $\mathrm{dt}$ \\
\hline RA & 12.35 & 3.49 & & 12.77 & 4.48 \\
$\mathrm{RV}$ & 12.35 & 3.88 & & 10.96 & 3.94 \\
RN & 7.53 & 4.08 & & 6.70 & 4.05 \\
Estrategias de & 51.19 & 8.56 & & 49.07 & 11.28 \\
Organización y & & & & \\
Comprensión & & & & \\
Autoeficacia (capacidad & 50.91 & 9.04 & & 49.01 & 10.63 \\
percibida) & & & & \\
Estrategias superficiales & 49.79 & 9.12 & & 50.01 & 10.65 \\
\hline
\end{tabular}

Por otra parte se ha utilizado una Su-bescala de la Escala REFEMA-57: es la de Estrategias de Aprendizaje y Autoeficacia (EAAE). Los 19 items de que consta esta sub-escala integra una solución factorial de tres dimensiones con una varianza explicada del $52.02 \%$, un coeficiente de adecuación muestral de 0.905 y un coeficiente de fiabilidad de 0.851 , lo que suponen unos coeficientes aceptables. La descripción de los factores es la siguiente:

Factor 1: Estrategias de organización y comprensión. Integran este factor 6 items y es una dimensión que evalúa estrategias de aprendizaje que inciden en los procesos de organización, comprensión y relación de contenidos cuando el alumnado aborda las tareas de estudio. Por ejemplo: "preparo los exámenes con atención y profundidad; repaso varias veces los temas hasta domninarlos completamente; utilizo técnicas para organizar como mapas o esquemas o para poner en orden los datos de los temas...; resumo las ideas más importantes de las lecciones para 
comprenderlas mejor...; tomo notas en las clases para recordar la información esencial..."

Factor 2. Autoeficacia, estrategias apoyo $y$ autorregulación aprendizaje. Integrada por 9 items, es una dimensión en la que se entrecruzan dos aspectos importantes en los procesos de estudio: la valoración de uno mismo como persona y como alumno y , a la vez, las estrategias de apoyo que sirven para dar seguridad al sujeto al afrontar las tareas de estudio. Como ejemplos podemos citar: "resisto las tareas de clase, aunque tenga dificultades; cuando me comparo con los demás me veo como un/a buen/a estudiante...; llevo mis tareas de clase al día; tengo confianza en mis habilidades...; me gustan los trabajos que me ponen las cosas y los temas difíciles...; creo que puedo aprender muchas cosas si me esfuerzo en mis trabajo personal...en la clase me encuentro relajado/a y puedo concentarme bien.. al hacer un examen me siento seguro/a de que lo haré bien..."

Factor 3: Estrategias superficiales y autoconcepto negativo. Está integrado por 3 items que responden a una forma negativa de encarar y abordar las tareas de estudio y aprendizaje. Por ejemplo: "nada más pensar en los exámenes me pongo nervioso/a...; durante los exámenes me cuesta concentrarme y sólo pienso en las consecuencias de hacerlos mal...; los exámenes me ponen nervioso/a y me quitan el sueño...”.

\section{Variables}

Se trata de la Escala BDPR (Almeida, 1988, 2003), compuesta por cinco subtests: Razonamiento Abstracto (RA), Razonamiento Verbal (RV), Razonamiento Numérico (RN), Razonamiento Espacial (RE) y Razonamiento Mecánico (RM). S e utilizan en este trabajo solamente las variables RA; RV y RN.

En cuanto a las Estrategias de aprendizaje se utilizan en este trabajo las siguientes: a) Organización $y$ comprensión de los recursos de estudio (tiempo, materiales) y preocupación en sistematizar la información y gestionarla adecuadamente; b) Autoeficacia, auto-regulación y estrategias de apoyo (percepción de de la capacidad para aprender con éxito e interés) y c) Estrategias superficiales y ansiedad ante exámenes que implican la ansiedad ante los exámenes con elevados niveles de tensión y preocupación ante las situaciones de evaluación.

\section{Procedimiento}

Los datos relativos a las variables estudiadas fueron recogidos por los autores de la investigación o por personal especialmente entrenado al efecto durante varias sesiones de trabajo en centros esolares públicos, pertenecientes a las ciudades de Porto, Braga y Chaves (Portugal) y A Coruña, Santiago de Compostela, Pontevedra y Ourense, en Galicia durante los años 2007 y 2008. Los estudiantes cumplimentaron de forma voluntaria y sin límite de tiempo los instrumentos de la investigación. Se les solicitaba la mayor objetividad posible al contestar y se les garantizaba la total confidencialidad de las respuestas. También se aclaraban todas las dudas que pudieran surgir durante las aplicaciones de las pruebas.

\section{Resultados y discusión}

Capacidad predictiva de las variables cognitivas de Razonamiento en interacción con las variables de Estrategias de aprendizaje

Tomando los resultados del análisis de regresión se verifica que el modelo es significativo en ambas las muestras de alumnado. Sin embargo, se comprueba que en la muestra del Norte de Portugal, el conjunto de variables que entran en la ecuación de regresión explica el $42.0 \%$ de la varianza total en el rendimiento escolar de los alumnos, quedando ese valor en apenas un $22.8 \%$ para la muestra de Galicia.

\section{Tabla 2.}

Resultados del Análisis de Regresión, método pasos sucesivos. VV. II.: Habilidades cognitivas de razonamiento numérico, verbal y abstracto. Autoeficacia y estrategias de aprendizaje de organización y comprensión y estrategias superficiales. V. D.: Rendimiento académico. (Ntotal= 785; 381 (Portugal) y 404 (Galicia, España) . Varianza total explicada: $42.0 \%$ (Portugal) y 22.8\% (Galicia, España)

\begin{tabular}{lcccccc}
\hline \multicolumn{1}{c}{$\begin{array}{c}\text { Variables } \\
\text { Predictoras }\end{array}$} & R & $\boldsymbol{R}^{2}$ & $\begin{array}{c}\text { Cambio } \\
\boldsymbol{R}^{2}\end{array}$ & $\begin{array}{c}\text { Beta } \\
\boldsymbol{\beta}\end{array}$ & $\begin{array}{c}\text { Sig. } \\
\boldsymbol{p}<\end{array}$ \\
\hline $\begin{array}{l}\text { Portugal } \\
1\end{array}$ & $\begin{array}{l}\text { Razonamiento numérico } \\
\text { (RN) }\end{array}$ & .497 & .247 & .247 & .497 & .000 \\
2 & $\begin{array}{l}\text { Autoeficacia (capacidad } \\
\text { percibida (AE) }\end{array}$ & .563 & .317 & .071 & .231 & .000 \\
3 & $\begin{array}{l}\text { Razonamiento verbal } \\
\text { (RV) }\end{array}$ & .601 & .361 & .073 & .250 & .000 \\
4 & $\begin{array}{l}\text { Estrategias de } \\
\text { organización y } \\
\text { comprensión (EOC) }\end{array}$ & .631 & .398 & .037 & .194 & .000 \\
$5 \begin{array}{l}\text { Estrategias superficiales } \\
\text { (ES) }\end{array}$ & .648 & .420 & .022 & -.154 & .000 \\
$\begin{array}{l}\text { Galicia/España } \\
1\end{array}$ & $\begin{array}{l}\text { Razonamiento numérico } \\
\text { (RN) }\end{array}$ & .407 & .166 & .166 & .407 & .000 \\
2 & $\begin{array}{l}\text { Autoeficacia (capacidad } \\
\text { percibida (AE) }\end{array}$ & .437 & .191 & .025 & .163 & .000 \\
3 & $\begin{array}{l}\text { Estrategias de } \\
\text { organización y } \\
\text { comprensión (EOC) }\end{array}$ & .452 & .205 & .013 & .107 & .000 \\
4 & $\begin{array}{l}\text { Razonamiento verbal } \\
\text { (RV) }\end{array}$ & .465 & .216 & .012 & .127 & .000 \\
5 Estrategias superficiales & .477 & .228 & .011 & -.114 & .012 \\
\hline
\end{tabular}

En ambas muestras hay cinco variables con una contribución significativa en el rendimiento académico. Estas cinco variables que entran en la ecuación son las mismas em ambas muestras, aunque en diferente orden, lo que nos lleva a pensar que son variables con una buena consistencia y que pueden ser relevantes en el conjunto de los sujetos de las muestras. Destacan las dos primeras variables tanto en la muestra de Portugal como en la de Galicia, ya que explican la mayor parte de la varianza: por una "Razonamiento numérico" y 
“Autoeficacia”. Véase que la varianza explicada para estas variables es de $31.8 \%$ de un total de $42 \%$ para la muestra de Portugal y el 19.1 de un total de $22.8 \%$. para la muestra de alumnado de Galicia (ver tabla 2).

Por otra parte, ya con menos importancia entran las tres variables restantes: "Estrategias de organización y comprensión”, "Razonamiento verbal” y "Estrategias superficiales/ansiedad ante exámenes”, resaltando el hecho de que esta última variable tiene una capacidad predictiva con un impacto en sentido negativo, lo que significa, en este caso, que alumnos con puntuaciones más elevadas en estrategias superificiales presentan con más intensidad un rendimiento académico bajo.

Destacar preferentemente la óptima capacidad predictiva que posee sobre el rendimiento la variable Razonamiento numérico en ambas muestras. Ello significa que a medida que los sujetos afronten sus tareas de estudio y aprendizaje mayor será el desarrollo y utilización de capacidades atencionales y habilidades de aprehensión y aplicación de relaciones conceptuales (razonamiento). Además se tiene muy en cuenta la especificidad de contextos o contenidos a los que se pueden aplicar tales habilidades de razonamiento

Los coeficientes obtenidos nos informan de que son las mismas variables las que intervienen en la explicación del rendimiento académico, tanto en el alumnado de Norte de Portugal como en el de Galicia, siendo igualmente cierto que el impacto de las mismas variables es positivo o es negativo en las dos muestras, pero es muy diferente el valor final de la varianza explicada en el rendimiento académico global. Hay que insistir que un $42 \%$ de la varianza total se observa en los alumnos portugueses, y un 23\% aproximado se obtiene en los alumnos de Galicia.

En cuanto a las estrategias de aprendizaje, estos resultados obtenidos permiten concluir que, la Autoeficacia/capacidad percibida, las Estrategias de organización y comprensión, son otras tantas variables que contribuyen a la explicación del buen rendimiento académico del alumnado. Coinciden estos datos, en buena parte, con otras investigaciones realizadas por A. Barca (Barca, 2009; Barca, Almeida, Porto, Peralbo y Brenlla, 2012) y otros autores como González-Pienda, J. A., Núñez, González-Pumariega, Alvarez, Roces, García, González, Cabanach y Valle (2000) y Valle, Cabanach, González-Pienda, Núñez, Rodríguez y Rosario (2009). Por el contrario, cuando dominan las Estrategias superficiales/ansiedad ante exámenes, el rendimiento académico disminuye. Creemos que este hecho ocurre, quizás, como consecuencia de un efecto de inhibición de la motivación de logro, tal como se puede observar a partir de las correlaciones negativas, y ello de acuerdo con otros trabajos recientemente realizados (Mascarenhas, 2004; Barca, Porto y Barca, 2009; González-Pienda, González-Cabanach, Núñez y Valle, 2002; Mascarenhas, Almeida y Barca, 2005).

Finalmente, estas Estrategias de aprendizaje del tipo de organización y comprensión, parece que configuran a los alumnos que optan por ellas como capaces de llevar a cabo las actividades de organización, comprensión y relación de contenidos al abordar las tareas de estudio.
Se anticipa que estos alumnos preparan los exámenes con atención y profundidad; repasan varias veces los temas hasta dominarlos, utilizan técnicas para organizar la información como mapas o esquemas o bien para poner en orden los diferentes contenidos..., en fin, se trata de hacer actividades que inciden positivamente en el aprendizaje y el buen rendimiento académico (Barca, Almeida, Porto, Peralbo y Brenlla, 2012)

\section{Referencias}

Ackerman, P. (1996). A theory of adult intellectual development: process, personality, interests, and knowledge. Intelligence, vol. 22, pp227-257.

Almeida, L.S. (1985). Os testes de racicionio diferencial em orientaçao vocacional. In J:F:A: Cruz, L.S. Almeida y O. F. Gonçalves (Eds.), Intervençao Psicologica na Educaçao. Porto: APLP.

Almeida, L. y Campos, B. (1986). Validade preditiva dos testes de raciocinio diferencial. Cadernos de Consulta Psicologica, vol. 2. pp. 105-118.

Almeida, L.S. (1988). O racicinio diferencial dos jovens: avaliaçao, desenvolvimento e diferenciaçao. Porto: Instituto Nacional de Investigaçao Científica.

Almeida, L.S. (1992). Inteligência e aprendizagem: dos seus relacionamentos à sua promoçao. Psicologia: Teoría e Pesquisa, vol. 8, pp-272-292.

Almeida, L. S., Antunes, A.M., Martins, T.B.O y Primi, R. (1997). Bateria de Provas de Raciocinio (BPR-5): Apresentaçao e procedimentos na sua construçao. Actas do I Congresso Luso-Espanhol de Psicologia da Educaçao. , 295-298.

Almeida, L.S. (1996), Cogniçao e aprendizagem: como a sua aproximaçao conceptual pode favorecer o desempenho cognitivo e a realizaçao escolar. Psicologia: Teoría e Pratica. vol 1, pp.17-32.

Almeida, L.S. (2003). Bateria de Provas de Raciocinio. Braga: Universidade do Minho/CIPsi.

Almeida, L. S., Antunes, A.M., Martins, T.B.O y Primi, R. (1997). Bateria de Provas de Raciocinio (BPR-5): Apresentaçao e procedimentos na sua construçao. Actas do I Congresso Luso-Espanhol de Psicologia da Educaçao. , 295-298.

Almeida, L.S. (1996), Cogniçao e aprendizagem: como a sua aproximaçao conceptual pode favorecer o desempenho cognitivo e a realizaçao escolar. Psicologia: Teoría e Pratica. vol 1, pp.17-32.

Almeida, L.S., Candeias, A., Primi, R., Ramos, C., Gonçalves, A.P, Coelho, H., Dias, J., Miranda, L. y Oliveira, E . P. (2003). Bateria de Provas de Raciocinio (BPR5-6. Estudo nacional de validaçao e aferiçao. Revista Psicologia e Eucaçao, 2 (1), 5-15.

Bandura, A. (1982). Teoría del aprendizaje social. Madrid: Espasa Calpe.

Bandura, A. (Ed.) (1999). Auto-Eficacia: como afrontamos los cambios de la sociedad actual. Bilbao: Desclée de Brouwer.

Barca, A., Brenlla, J. C., Santamaría, S. y A. González (1999): Estrategias y enfoques de aprendizaje, contextos familiares y rendimientro académico en el alumnado de educación secundaria. Indicadores para un análisis causal. Revista Galego-Portuguesa de Psicoloxía e Educación., 3 (vol. 4) 229-272. 
Barca, A., Marcos, J.L., Núñez, J.C., Porto, A. y R. Santorum (1997). Procesos de aprendizaje en ambientes educativos. Madrid: Centro de Estudios Ramón Areces.

Barca, A., Porto, A., Santorum, R., Morán, H. y Brenlla, J.C. (2008). Los procesos y estrategias de aprendizaje en alumnado universitario. Un análisis comparativo entre las Escalas ACRA y el Cuestionario CEA. Oviedo: Actas del $V^{o}$ Congreso Internacional de Psicología y Educación. Pp. 640-669.

Barca, A., Porto, A. y Barca, E. (2009). Motivación y aprendizaje en el alum-nado de educación secundaria con alto y bajo rendimento académico: um análisis desde la diversidad e inclusión educativa. En Barca, A. (2009) (Coord.). Motivación y aprendizaje en contextos educativos. Granada: Grupo Editorial Universitario.

Barca, A. (Coord.) (2009). Motivación y aprendizaje en contextos educativos. Granada: Grupo Editorial Universitario.

Barca, A., Almeida, L.S., Porto, A., Brenlla, J.C. Barca, E., Morán; H. (2010). Impacto de las metas académicas y estrategias de aprendizaje en el rendimiento académico. Oviedo: VII Congreso Iberaomericano de Psicología.

Barca, A. Almeida, L., Porto, A., Peralbo, M. y Brenlla, J. C. (2012). Motivación escolar y rendimiento: impacto de metas académicas, estrategias de aprendizaje y autoeficacia. Anales de Psicología, vol. 28 (3), pp. 848-859.

Barca, A., Almeida, L., Porto, A., Peralbo, M, y Brenlla, J.C. (2012). Motivación escolar y rendimiento: impacto de metas académicas, de estrategias de aprendizaje y autoeficacia. Anales de psicología, Vol. 28, 3, 848-859.

Beltrán, J. (1993). Procesos, estrategias y técnicas de aprendizaje. Madrid: Síntesis.

Beltrán, J. A., Pérez, L. F. y Ortega, M. I. (2006). CEA. Cuestionario de Estrategias de Aprendizaje. Madrid: TEA.

Brenlla, J. C. (2005). Atribuciones causales, enfoques de aprendizaje, rendimiento académico y compretencias bilingües en alumnos de educación secundaria. Un análisis multivariable. A Coruña: Universidad de A Coruña (tesis doctoral, inédita).

Cano, F. (1994). Estrategias, metaestrategias y estilos de aprendizaje. En M.V. Trianes (Ed.), Psicología de la Educación para profesores. Madrid: Pirámide.

Cano, F. y Justicia, F. (1993). Factores académicos, estrategias y estilos de aprendizaje. Revista de Psicología General y Aplicada, 46, 89-99.

Cano, F. y Justicia, F. (1994). Learning styles, strategies and approaches: an analysis of their interrelationships. Higher Education, 27, 239-260.

Cattell, R. B . (1971). Abilities: their Structure, Growth and Action. Boston: Houghton-Mifflin.

Ceci, S. (1991). How much does schooling influence general intelligence and its cognitive components? A reassessment of the evidence. Developmental Psycholoy, vol. 27, pp. 703-722.

Dansereau, D.F. (1985). Learning strategy research. In S.F. Chipman, J.W. Segal y R. Glaser (Eds.). Thinking and learning skills, Vol. I. Relating instruction to research. Hillsdale, NJ: Lawrence Erlbaum Associates.

De la Fuente, J. (2004). Perspectivas recientes en el estudio de la motivación: la teoría de la orientación de metas. Revista Electrónica de Investigación Psicoeducativa, 2 (1), 35-62.

García, T., y Pintrich, P.R. (1994). Regulating motivation and cognition in the classroom: The role of self-schemas and self-regulatory strategies. En D.H. Schunk, y B.J. Zimmerman (eds.). Self-regulation of learning and performance. Issues and educational applications. Hillsdale, NJ.: LEA.

Gardner, H. (1995). Inteligencias múltiples. La teoría en la práctica. Barcelona: Paidós. (Orig. 1993).

Gargallo, B. (2000). Estrategias de aprendizaje. Un programa de intervención para ESO y EPA. Madrid: Ministerio de Educación, Cultura y Deporte. Centro de Investigación y Documentación Educativa.

Garma, A. M ${ }^{\mathrm{a}}$ y Elexpuru, I. (1999): El autoconcepto en el aula. Barcelona: EDEBE.

González-Pienda, J. A., Núñez, J. C., GonzálezPumariega, S., Alvarez, L., Roces, C., García, M., González, P., Cabanach, R. y Valle, A. (2000). Autoconcepto, proceso de atribución causal y metas académicas en niños con y sin dificultades de aprendizaje. Psicothema, vol. 12, 4, 548-558.

Hernández, P. y García, L. (1991). Psicología y enseñanza del estudio. Madrid: Pirámide.

Lemos, G., Almeida, L. Guisande, A. y Primi, R. (2008). Inteligência e rendimento escolar: análise da sua relaçao ao longo da escolaridade. Revista Portuguesa de Educaçao, 21 (1), pp. 83-99.

Mascarenhas, S. (2004). Avaliaçao dos processos, estilos e abordagens de aprendizagem dos alunos do ensino médio do Estado de Rondônia (Brasil). A Coruña: Universidade da Coruña (Tesis doctoral. Inédita).

Mascarenhas, S., Almeida, L. S. y Barca, A. (2005a). Atribuições causais e rendimento escolar: Impacto das habilitaçoes escolares dos pais e do género dos alunos. Revista Portuguesa de Educação, 18, 77-91.

Mascarenhas, S., Almeida, L. S. y Barca, A. (2005b). Estilos atribucionais e rendimento académico: Um estudo com estudantes brasileiros do ensi-no médio. Revista Galego-Portuguesa de Psicoloxía e Educación, 10, 221-228.

Nisbet, J. y Shucksmith, J. (1987). Estrategias de aprendizaje. Madrid: Santillana.

Núñez, J.C. y J.A. González-Pienda (1994). Determinantes del rendimiento académico. Oviedo: SPU.

Primi, R. y Almeida, L. (2000). Estudo de validaçao da Bateria de Provas de Raciocionio (BPR-5). Psicologia: Teoria e Pesquisa, vol 16, n 2, pp. 165173.

Román, J. M. y Gallego, S. (1991). Escala de Estrategias de Procesamiento de Información. Universidad de Barcelona. III Congreso de Evaluación Psicológica.

Román, J. M. y Gallego, S. (1997). ACRA: Escalas de Estrategias de Aprendizaje. Madrid: TEA.

Schunk, D.H. (2000). Learning Theories: An Educational Perspective. New Jersey: Prentice Hall. 
Valle, A., Cabanach, R., González-Pienda, J. A., Núñez, J. C., Rodríguez, S. y Rosário, P. (2009). Perfiles motivacionales en estudiantes de secundaria: análisis diferencial en estrategias cognitivas, estrategias de autorregulación y rendimiento académico. Revista Mexicana de Psicología, Vol. 26, 1, 113-124 